University for Business and Technology in Kosovo

UBT Knowledge Center

UBT International Conference

2015 UBT International Conference

Nov 7th, 9:00 AM - 5:00 PM

\title{
Perfect Metamaterial absorber based energy harvesting application in ISM Band
}

\author{
Furkan Dincer \\ Aralik University, furkandincer@kilis.edu.tr \\ Mehmet Bakir \\ Iskenderun Technical University \\ Muharrem Karaaslan \\ Iskenderun Technical University \\ Kemal Delihacioglu \\ Aralik University \\ Cumali Sabah \\ METU
}

Follow this and additional works at: https://knowledgecenter.ubt-uni.net/conference

Part of the Computer Sciences Commons, and the Digital Communications and Networking

Commons

\section{Recommended Citation}

Dincer, Furkan; Bakir, Mehmet; Karaaslan, Muharrem; Delihacioglu, Kemal; and Sabah, Cumali, "Perfect Metamaterial absorber based energy harvesting application in ISM Band" (2015). UBT International Conference. 91.

https://knowledgecenter.ubt-uni.net/conference/2015/all-events/91

This Event is brought to you for free and open access by the Publication and Journals at UBT Knowledge Center. It has been accepted for inclusion in UBT International Conference by an authorized administrator of UBT Knowledge Center. For more information, please contact knowledge.center@ubt-uni.net. 
International Conference on Computer Science and Communication Engineering, Nov 2015

15. Kolaczyk, E. D., Csárdi, G.: Statistical Analysis of Network Data with R, Use R! 65, DOI: 10.1007/978 - 1 - 4939 - 0983 - 4_4, Springer Science + Business Media, New York (2014)

\title{
Perfect Metamaterial absorber based energy harvesting application in ISM Band
}

\author{
Furkan Dincer $^{1}$, Mehmet Bakir ${ }^{2}$, Muharrem Karaaslan ${ }^{2}$, Kemal Delihacioglu ${ }^{1}$, Cumali \\ $\mathrm{Sabah}^{3}$ \\ ${ }^{1}$ Department of Electrical and Electronics Engineering, Kilis 7 Aralik University, Kilis, \\ Turkey \\ ${ }^{2}$ Department of Electrical and Electronics Engineering, MTMs and Photonics Research \\ Group Iskenderun Technical University, Hatay, Turkey \\ ${ }^{3}$ Department of Electrical and Electronics Engineering, METU - Northern Cyprus \\ Campus TRNC / Mersin 10, Turkey \\ furkandincer@kilis.edu.tr
}

\begin{abstract}
An electromagnetic (EM) energy harvesting application based on metamaterial absorber (MA) is introduced and discussed in this paper. This application is operating at ISM band $(2.40 \mathrm{GHz})$ which is especially chosen due to its wide usage area. Square ring resonator (SRR) which has two gaps and resistors across the gaps on it is used. Chips resistors are used to deliver power to any active component. Transmission and reflection characteristics of metamaterial absorber (MA) for energy harvesting application are investigated. $83.6 \%$ efficient for energy harvesting application is realized in this study.
\end{abstract}

Keywords: Metamaterials, Absorber, Energy harvesting

\section{Introduction}

Metamaterials (MTMs), due to their exotic application areas attract attention in recent years. These applications can be categorized as follows. Cloaking [1], antennas [2], sensors [3-4], absorbers [5, 6], etc. [7-8].Nowadays, new applications by using split ring resonators (SRRs) in MTMs came out [910]. When electromagnetic waves excite SRRs, generated electromagnetic energy is absorbed by resonators. This energy can be used as a voltage source, In order to harvest the energy, a gap is positioned on SRR coherent with the electric field direction and lumped element is placed on a gap to harvest this energy. Lumped element can optionally be a varicap or resistor. This mechanism is a basis for energy harvesting applications. If a lumped element is placed across the gap such a varicap, tunable harvesting applications can be possible. In this study, the lumped elements are choosen as resistors which are the recipient of the harvested energy. Resistance value is determined according to the numerical results. EM Energy harvesting concept is new technology for MTM science, but it has big importance due to limited energy resources and big field of applications.

Power harvesting devices are generally used to convert one type of energy as acoustic or optical to another type of energy which is generally DC [11-12]. Though EM MTMs provide flexible design options and they can be adapted to various energy harvesting studies, there is few electromagnetic energy harvesting studies with MTMs in current literature [13-15]. At first, $611 \mathrm{mV}$ voltage is harvested at $5.8 \mathrm{GHz}$ by using electromagnetic MTMs[13]. Energy conversion by using 3-D metamaterial arrays are presented in [14], at [15], 36.8\% power efficiency is experimentally obtained at $900 \mathrm{MHz}$.

This study has different sides and advantages from the others as explained below. First advantage of this study is having simple geometry. Simple geometry means simple production, anyone who wants to produce this MTM energy harvester can produce easily. Other advantage is that; this study based on metamaterial absorber (MA) together with tunability option. It is possible to get more than $83.6 \%$ 
efficient MTM energy harvester with this option. Other advantage of this study is the realization for ISM band. Lots of appliances are operated at this band so it can be possible to harvest energy without making extra transmitters. The organization of this study is as follows. In Section 2, the structure geometry based on SRR is proposed, and the numerical method is presented. In Section 3, numerical results are presented. Finally, summary and conclusions are provided and discussed in Section 4.

\section{Design and Numerical}

In this study, a SRR as an example of resonant MTM particle is used as a MA based energy harvester. Square ring resonators (SRR) used in this design have two $7.2 \mathrm{~mm}$ gaps. Resistors are placed as lumped network elements across the gaps on SRR. Resistance value is chosen as $2000 \Omega$ which will be explained later. FR4 material is chosen as a substrate to support resonator and reduce resonance frequency as well as frequency selective surfaces. FR4 substrate has a thickness of $1.6 \mathrm{~mm}$ with loss tangent and relative permittivity of FR4 0.02 and 4.2, correspondingly. Copper is used as metallic element with a thickness of $0.035 \mathrm{~mm}$ and it has a conductivity of $5.8 \times 10^{8} \mathrm{~S} / \mathrm{m}$. An air gap which has a thickness of $4.2 \mathrm{~mm}$ is placed behind the substrate. Back of dielectric-air gap, copper plate is placed as an exact reflector to block signal transition. Copper plate and air gap dimensions are hold same with FR4 substrate dimensions. Design details can be seen in Fig. 1. Numerical results are achieved by commercially available full-wave electromagnetic solver which uses finite integration technique.

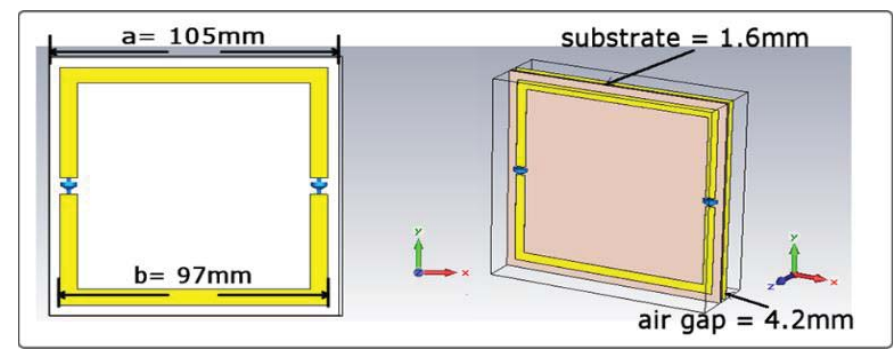

Fig. 1. Dimensions of the MA for energy harvesting study.

The frequency response of absorber can be calculated by $\mathrm{A}(\omega)=1-\mathrm{R}(\omega)-\mathrm{T}(\omega)$, where $\mathrm{A}(\omega), R(\omega)$ and $T(\omega)$ define the absorption, reflectance, and transmittance, respectively. When reflection $R(\omega)=\left|S_{11}\right|^{2}$ and transmission $T(\omega)=\left|S_{21}\right|^{2}$ falls to minimum values, absorption rises to maximum at the resonance frequency. There will be no transmission to be examined since a metal plate is assigned behind air type dielectric.

The perfect absorption happens when the reflection is close to zero. When perfect absorption is observed power harvested from resistors rises which is crucially important for energy harvesting studies. It is possible to reduce reflection to near-zero by choosing the relative effective permittivity $\varepsilon(\omega)$ and permeability $\mu(\omega)$ values closer to provide impedance matching with air. It is possible to absorb both the incident electric and magnetic field by properly tuning $\varepsilon(\omega)$ and $\mu(\omega)$ of the effective medium of absorber. There are some studies in literature to explain absorption mechanism of the structures [15-18].

\section{Results and Discussion}

In order to show the performance of the proposed MA based energy harvester, numerical study is realized and assessed. Numerical values of reflection and absorption are given in Fig. 2. It is seen from Fig. 2 that the absorption and reflection values are $99.99 \%$ and $0.02 \%$, respectively when $2000 \Omega$ resistors are placed in gaps. According to these values suggested MA structure shows perfect absorption features at $2.40 \mathrm{GHz}$. 


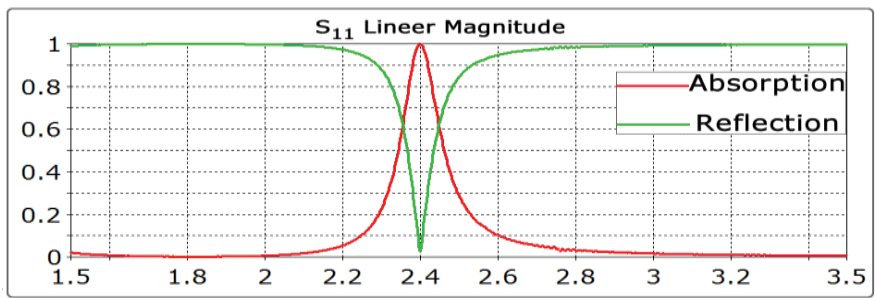

Fig. 2. Absorption and reflection value.

One of the most important feature of power harvesters is RF to DC power conversion. Power conversion can be calculated as [13];

$$
\rho=\frac{P_{D C}}{P_{R F}}
$$

$P_{R F}$ isdefined as $1 W$ by simulation program manufacturer. $P_{D C}$ values are extracted from voltage and current values of resistors used in this study.Resistors are placed across the gaps of SRR to obtain and easily convert voltage value using conventional methods. The resistance value has a critical importance and a great effect on the total energy conversion efficiency of the structure. In order to show the harvested power performance for MA based harvesting application, the effects of resistance value on power harvesting efficiency is investigated for comparison. This comparison enables a deeper understanding for operating mechanism of the suggested structure. The efficiency is examined for ten different resistance values between $500 \Omega$ and $5.000 \Omega$ Numerical results are given in Fig. 3 .

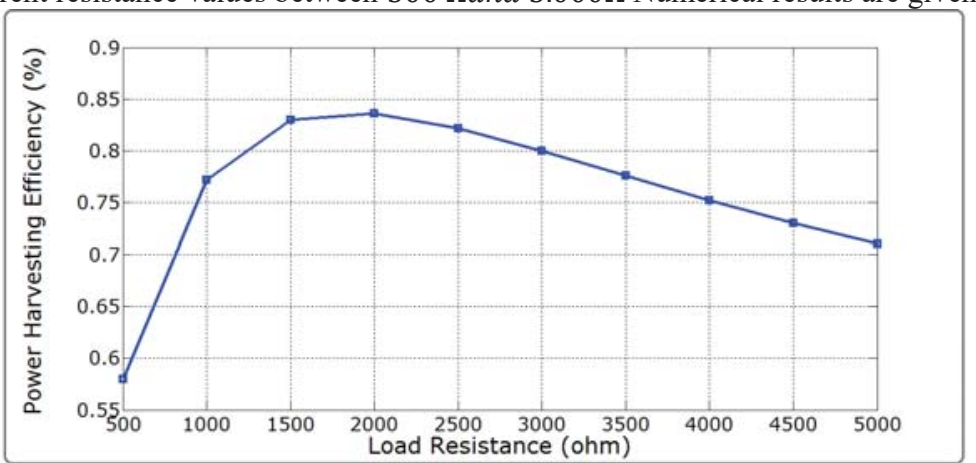

Fig. 3. RF to DC efficiency of SRR according to load resistance

As shown in Fig. 3, changing the resistance values between $500 \Omega$ to $2.000 \Omega$ results in significant change in power harvesting efficiency between 58\% to 83.6\%. When $2 \mathrm{k} \Omega$ resistance is used, $0.418 \mathrm{~W}$ power is obtained from a single resistor which means $0.836 \mathrm{~W}$ power collection by using two resistors. For this reason overall efficiency of MA energy harvester becomes around $83.6 \%$ according to the equation of $\frac{P_{D C}}{P_{R F}}$. This is the highest energy harvesting value in the literature. Beside this, the efficiency of the proposed structure linearly changes depending on the load resistance $2 \mathrm{k} \Omega-$ $5 k \Omega$. Hence the system can also be adopted to many sensor applications of which resistance changes linearly with respect to the intended measurement parameters.

Table 1. Energy Harversting Efficiency Comparison Table (Single Array)

\begin{tabular}{|l|l|}
\hline Reference study & Efficiency (\%) \\
\hline Ramahi [13] & $76 \%$ \\
\hline Almoneef [14] & $68 \%$ \\
\hline Hawkes [15] & $65 \%$ \\
\hline Proposed Study & $83.6 \%$ \\
\hline
\end{tabular}


Metal plate which is behind the air gap is acting important role in MA based harvesting studies. EM energy is confined between metal plate and resonator which increases the power across the resistors. A numerical application is realized and investigated for the proposed MA based energy harvesters. Two different configurations (with-metal plate and without-metal plate) are created and investigated for resistance value of $2 k \Omega$. First investigation for this numerical setup is related with absorption rates. As it seen from Fig.4, when metal plate is present behind the air gap, absorption value becomes $99.99 \%$ at $2.40 \mathrm{GHz}$. In contrast to the first model, when metal plate is not used, absorption value drops dramatically to $44 \%$ at $2.46 \mathrm{GHz}$.

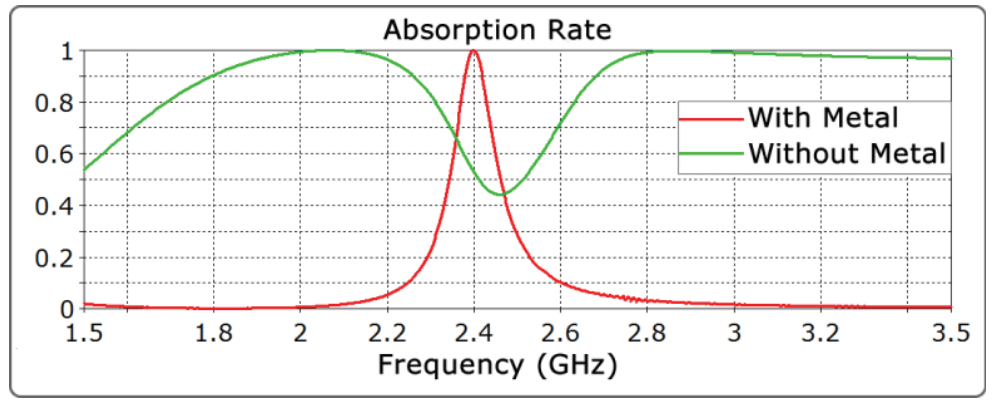

Fig. 4. Absorption Rate of MA base energy harvesting studies according to existence of metal plate.

Second investigation is related with power and resonance frequency according to the existence of metal plate. For the first case as it seen from Fig. 5 that power value which is harvested from a single resistor is $0.418 \mathrm{~W}$ at $2.40 \mathrm{GHz}$. However it is $0.11 \mathrm{~W}$ at $2.46 \mathrm{GHz}$ for the without-metal plate model. Metal plate affects the resonance frequency and absorption level directly because it creates EM effects. It can be seen that the position of the resonance is shifted from $2.40 \mathrm{GHz}$ to $2.46 \mathrm{GHz}$ when metal plate is removed. This means that each case has a different characteristic, capacitive effect and power level. Also, the metal plate keeps the EM transmission value near zero. It is concluded from this numerical analysis that adding metal plate in MTM energy harvesting studies increases the power harvesting efficiency in high amounts. As shown in the table 1, when this work is compared with other studies in current literature, efficiency reaches maximum value in this study. Hence, MTM based harvester is a novel and challenging study in literature with its better results and primness.

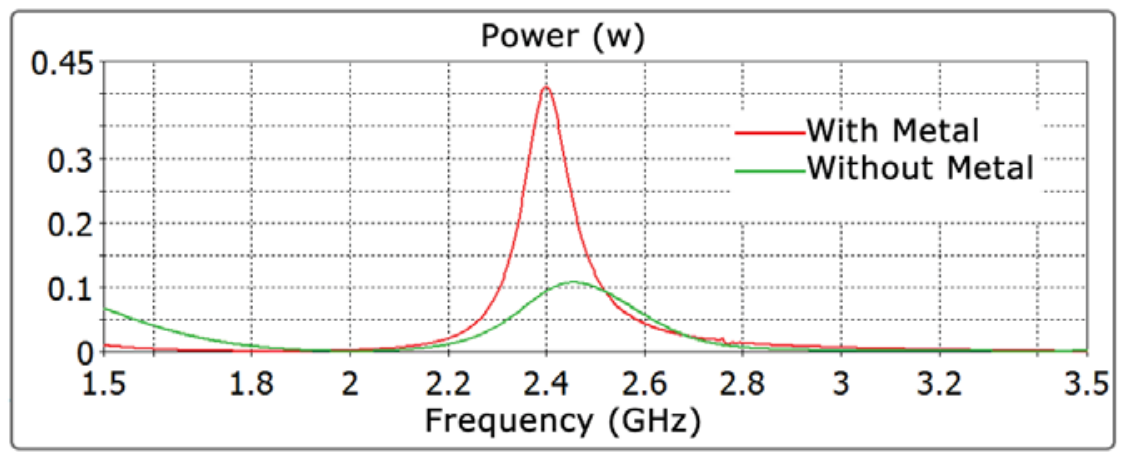

Fig. 5. Power harvested across the lumped element according to existence of metal plate.

Physical mechanism and operation principle can be understood easily according to electric field distributions of front and side metallic parts of the absorber. Electric field distributions are given at the resonance frequency of $2.40 \mathrm{GHz}$. It can be seen from Fig.6. that the electric field is concentrated on SRR and it is confined due to metal plate. By adding a resistor to the resonator, the structure can be modeled as a voltage source. In order to realize this, structure is modeled as perfect absorber which provides maximum EM energy harvesting at the resonance frequency. 


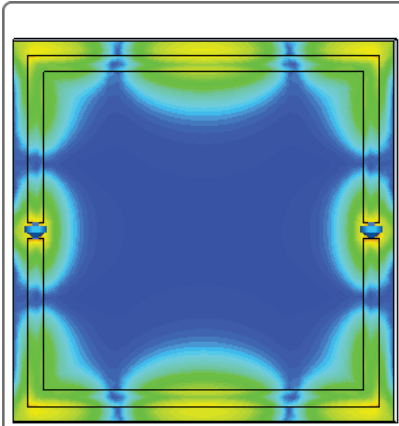

Front Side

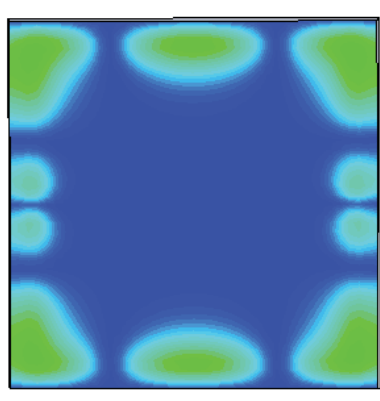

Back Side

Fig. 6. Electric field distribution at resonance frequency.

\section{Conclusion}

In this study, an energy harvesting application operating at ISM band is proposed and discussed. Electromagnetic characteristics of the proposed design and usage areas in EM energy harvesting applications are discussed. Efficient pPower harvesting with $83.6 \%$ efficient at $2.40 \mathrm{GHz}$ is demonstrated numerically. Numerical results prove that this study can be used in energy harvesting application efficiently. As a result, proposed structure can be used as MA based energy harvester with high efficiency in ISM band.

\section{References}

1. Pendry, J. B., Schuriand, D., Smith D. R.: Controlling electromagnetic fields Science, Vol. 312. (2006) 1780-1782

2. Li, D., Szabo, Z., Qing, X., Li, E. P., Chen, Z. N.: A high gain antenna with an optimized metamaterial inspired superstrate IEEE T. Antenn. Propag, Vol. 60. (2012) 6018-6023

3. Sabah, C., Dincer, F., Karaaslan, Unal, M. E., Akgol, O., Demirel, E.: Perfect metamaterial absorber with polarization and incident angle independencies based on ring and cross-wire resonators for shielding and a sensor application Opt. Commun, Vol. 322 (2014) 137-142

4. Shelby, R.A., Smith, Schultz, D.R.:Experimental verification of a negative index of refraction, Vol. 292. Science (2001) 77-79

5. Chen, H.T.: Interference theory of metamaterial perfect absorbers, Opt. Express, Vol. 20. (2012) 7165-7172

6. Lee, J., Lim, S.: Bandwidth-enhanced and polarization-insensitive metamaterial absorber usingdouble resonance Electron. Lett, Vol.47 (2012) 8-9.

7. Esen, M. Ilhan, I., Karaaslan, M., Unal, E., Dincer, F., Sabah, C.: Electromagnetic absorbance properties of a textile material coated using filtered arc-physical vapor deposition method, J. Ind. Text, (2014) 1528083714534710

8. Dincer, F., Karaaslan, M. Unal, E. Akgol, O., Demirel, E., Sabah, C.:Polarization and angle independent perfect metamaterial absorber based on discontinues cross-wire-strips,"J. Electromagn. Waves Appl, Vol. 28. (20149 741-751.

9. Lee, H.J., Yook, J.G.:Biosensingusingsplit-ring resonator at microwaveregime, Appl. Phys.Lett.,Vol.92. (2008) 254103:1-254103:3

10. Ou, J.Y., Plum, E., Jiang, L., Zheludev, N.I.: Reconfigurablephotonic metamaterials, Nano Lett, Vol.11. (2011) 2142-2144,

11. Lallart, M., Guyomar, D. Richard C., Petit, L.: Nonlinear optimization of acoustic energy harvesting using piezoelectric devices, J. Acoust. Soc. Am., Vol.128. (2010) 2739-2748

12. Zhu, N., Ziolkowski, R. W., Xin, H.: A metamaterial-inspired, electrically small rectenna for high-efficiency low power harvesting and scavenging at the GPS L1 frequency, Appl. Phys. Lett., (2011) Vol. 99. 114101 
13. Ramahi,O. M., Almoneef, T. S., AlShareef, M., Boybay, M. S.:Metamaterial particles for electromagnetic energy harvesting, Appl. Phys. Lett. (2012) Vol. 101. 173903

14. Almoneef, T. S., Ramahi, O. M.: A 3-dimensional stacked metamaterial arrays for electromagnetic energy harvesting, Prog. Electromagn.Res., (2014) Vol. 146. 109-115

15. Hawkes, A. M., Katko, A. R., . Cummer, S. A.: A microwave metamaterial with integrated power harvesting functionality, Appl. Phys. Lett., Vol. 103. (2013) 163901

16. Dincer,F., Akgol,O., Karaaslan, M., Unal, E., Sabah, C.: Polarization angle independent perfect metamaterial absorbers for solar cell applications in the microwave, infrared, and visible regime, Prog. Electromagn.Res, Vol. 144. (2014) 93-101,

17. Sabah, C., Dincer, F., Karaaslan, M., Unal, E., Akgol, O.: Polarization-Insensitive FSS based Perfect Metamaterial Absorbers in GHz and THz Frequencies, Radio Science, Vol.49. (2014) 306-314

18. Dincer, F., Karaaslan, M., Unal, E., Akgol, O., Sabah, C.: Design of Polarization- and Incident Angle-Independent Perfect Metamaterial Absorber with Interference Theory, Journal of Electronic Materials, Vol. 43. (2014) 3949-3953 